\title{
A Study on Bio-Compatible Piezoelectric Materials by First Principles Calculation*
}

\author{
Yasutomo UETSUJI ** Kouhei IMOTO ** Sadaomi KUMAZAWA ** \\ Kazuyoshi TSUCHIYA *** Sei UEDA ** and Eiji NAKAMACHI ** \\ ** Department of Mechanical Engineering, Osaka Institute of Technology \\ 5-16-1 Omiya, Asahi-ku, Osaka, Japan \\ E-mail:uetsuji@med.oit.ac.jp \\ *** Department of Precision Engineering, Tokai University \\ 1117 Kitakaname, Hiratsuka, Kanagawa, Japan
}

\begin{abstract}
Bio-compatible piezoelectric materials are becoming increasingly important for actuators and sensors in medical devices, that is Bio-MEMS such as health monitoring systems and drag delivery systems. In this study, we challenged to derive new piezoelectric materials with bio-compatibility by first principles calculation. Firstly, constituent elements of bio-compatible piezoelectric materials have been specified by HSAB method from the viewpoint of interaction energy with in-vivo molecules. Secondly, in order to create a perovskite-type crystal structure with good piezoelectric response, the combination of bio-compatible elements was selected to satisfy geometric stable condition defined by tolerance factor. As a result, we discovered 7 kinds of new piezoelectric materials. We focused on one of them, $\mathrm{MgSiO}_{3}$ that is known to be a mineral with perovskite-type crystal structure, and analyzed the stable cubic structure at paraelectric non-polar phase and the stable tetragonal structure at ferroelectric phase by first principles DFT. Additionally, structural phase transition of $\mathrm{MgSiO}_{3}$ has been investigated on the assumption of linear structural change from cubic structure to tetragonal one. DFT calculation indicated that $\mathrm{MgSiO}_{3}$ can change spontaneously to tetragonal structure with high tetragonality and polarization, and that $\mathrm{MgSiO}_{3}$ can present a good piezoelectricity.
\end{abstract}

Key words: Computational Mechanics, Biomaterials, Piezoelectric Ceramics, HSAB Method, Density Functional Theory, Structural Phase Transition

\section{Introduction}

Nowadays, piezoelectric materials are becoming increasingly important for actuators and sensors in medical devices, that is Bio-MEMS such as health monitoring systems and drag delivery systems. Actuators and sensors for medical devices are required not only high piezoelectricity but also good bio-compatibility because their constituent elements may dissolve into body fluid and attack in-vivo molecules. On the other hand, perovskite-type compounds, that are lead zirconium titanate ( $\mathrm{PZT}: \mathrm{Pb}(\mathrm{Zr}, \mathrm{Ti}) \mathrm{O}_{3}$ ) ceramics with the highest piezoelectricity, have been used for actuators and sensors as a component of various electronic and mechanical devices. Additionally, PZT piezoelectric ceramics are applied to new technological fields such as micro/nano machines ${ }^{(1)}$. However, PZT piezoelectric ceramics include hazardous lead ( $\mathrm{Pb}$ ), therefore they can not be applied to medical devices. WEEE (Waste Electrical and Electronic Equipment) ${ }^{(2)}$ and RoHS (Restriction on Hazardous Substances) ${ }^{(3)}$ were approved in Europe, and the use of hazardous substances

\footnotetext{
${ }^{\text {*Received }} 19$ Dec., 2006 (No. T-04-0417) Japanese Original : Trans. Jpn. Soc. Mech. Eng., Vol.71, No.706, A (2005), pp.944-951 (Received 12 Apr., 2004) [DOI: 10.1299/jmmp.1.191]
} 
was restricted strongly. Consequently, lead-free piezoelectric materials such as bismuth layer-structured ferroelectrics $\left(\mathrm{SrBi}_{2} \mathrm{Ta}_{2} \mathrm{O}_{9}\right){ }^{(4)}$ and potassium niobate $\left(\mathrm{KNbO}_{3}\right)^{(5)}$ are researched energetically. However, there is incomplete understanding of their bio-compatibility since they are aimed for the application to electrical and electronic equipments except medical devices. Furthermore, the hitherto material study is focused on improvement of existing lead-free materials or substitution of lead with other elements, it is lacking in progressive search for a quite new material.

In this study, new lead-free perovskite-type compounds are derived systematically by first principles calculations for the purpose of developing a new bio-compatible piezoelectric material for medical devices. This study is focused on the following three points; (1) Establishment of bio-compatibility standards set by HSAB (Hard Soft Acids and Bases) principle (6) and specification of bio-compatible elements. (2) Research of bio-compatible elementary combination for perovskite-type compound. (3) First principles evaluations of stable structure and material characteristics for cubic and tetragonal crystal structures.

\section{First Principles Approach for New Bio-Compatible Piezoelectric Materials}

The important target of promising piezoelectric materials is perovskite-type compounds $\mathrm{ABX}_{3}$. As shown in Fig.1, new bio-compatible perovskite-type compounds are researched by the following two steps: [I] specification of bio-compatible elements and [II] elementary combination and crystal structure evaluations for perovskite-type compounds. In the first step [I], all of industry-useful metal elements are classified into 3 kinds, toxicity elements, bio-essential elements and the others. Toxicity elements are excluded, while bio-essential elements are considered into bio-compatible elements. And then, if the others except toxicity and bio-essential elements have low interaction energy with in-vivo molecules, they are also added to bio-compatible elements. In the second step [II], we employ bio-compatible elements for $\mathrm{A}$ and $\mathrm{B}$, oxygen for $\mathrm{X}$ in perovskite-type compound $\mathrm{ABX}_{3}$. At first, stable combinations of A, B and X elements are searched by their ion radiuses. Next, potential energy of stable combinations is calculated, and cubic and tetragonal crystal structures are evaluated by DFT (Density Functional Theory). Finally, material characteristics are estimated for the obtained crystal structures.

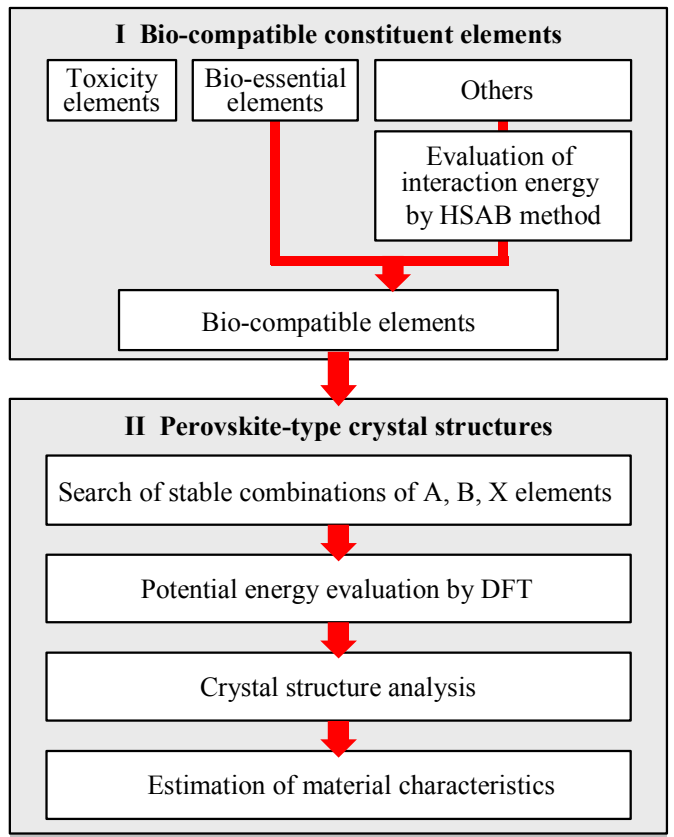

Fig.1 Flow chart of first principles study on bio-compatible piezoelectric materials. 


\subsection{Selection of bio-compatible constituent elements}

After piezoelectric materials are dissolved into metal ions in body fluid, they may have the following influence on a living body: (a) Destruction of biological functions caused by bonging with proteins. (b) Genetic damage caused by reaction with DNA (deoxyribonucleic acid). (c) Biological lesion caused by substitution of bio-essential elements. In this study, substituting and competing elements $(\mathrm{Pb}, \mathrm{Tl}, \mathrm{Cd}, \mathrm{Sr}, \mathrm{W})$ and catalytic toxicity elements $(\mathrm{Hg}$, $\mathrm{Cs}, \mathrm{Ru}, \mathrm{Rh}, \mathrm{Pd}, \mathrm{Os}, \mathrm{Ir}, \mathrm{Pt}, \mathrm{Co}$ ), that are classified into type (c), are excluded from constituent elements. On the other hand, the bio-compatibility is estimated from the interaction energy for metal elements ( $\mathrm{Au}, \mathrm{Ba}, \mathrm{Be}, \mathrm{Bi}, \mathrm{In}, \mathrm{Li}, \mathrm{Rb}, \mathrm{Re}, \mathrm{Sc}, \mathrm{Tc}, \mathrm{Y}$ ) that may have the influence such as types (a) and (b). Furthermore, bio-essential elements $(\mathrm{Ca}, \mathrm{Cr}$, $\mathrm{Cu}, \mathrm{Fe}, \mathrm{Ge}, \mathrm{Mg}, \mathrm{Mn}, \mathrm{Mo}, \mathrm{Na}, \mathrm{Ni}, \mathrm{Sn}, \mathrm{V}, \mathrm{Zn}$ ) and already-known in-vivo elements ( $\mathrm{Si}, \mathrm{Ca}$, $\mathrm{Ta}, \mathrm{Ti}, \mathrm{Zr}$ ) are added to constituent elements with no evaluation of interaction energy.

\subsection{Bio-compatibility evaluation by using HSAB principle}

Metal ions dissolved from piezoelectric materials are in danger of biological and genetic damage caused by reaction with in-vivo molecules such as proteins and DNA. The reaction between metal ions and in-vivo molecules can be reviewed as a chemical reaction between a Lewis acid and a Lewis base. Consequently, in order to evaluate the reactivity of metal ions on proteins and DNA, we employ the HSAB principle which states that "hard acids prefer to coordinate to hard bases and soft acids to soft bases" ${ }^{\prime(6)}$. The hardness or softness (inverse of hardness) of species can be defined quantitatively by using the idea of polarizability. A less polarizable species is hard and a more easily polarized one is soft. As an indicator of reactivity, HSAB principle presents the interaction energy between acid and base which is defined by each electronegativity and hardness. In this study, the interaction energy between metal ions and important in-vivo molecules is evaluated quantitatively by HSAB principle, and little-reactionary ions are distinguished into bio-compatible elements.

The absolute electronegativity $\chi_{\mathrm{S}}$ of a neutral species $\mathrm{S}$ is defined by the energy $E_{\mathrm{S}}$ and the number of electron $N_{\mathrm{S}}$, and its negative is equal rigorously to the chemical potential of electrons $\mu_{\mathrm{S}}$ as shown in Eq.(1).

$$
\chi_{\mathrm{s}}=-\left(\frac{\partial E_{\mathrm{S}}}{\partial N_{\mathrm{S}}}\right)=-\mu_{\mathrm{S}}
$$

Additionally, the absolute electronegativity $\chi_{\mathrm{S}}$ can be also expressed by Eq.(2) if ionization potential $I_{\mathrm{S}}$ and electron affinity $A_{\mathrm{S}}$ are given for the species $\mathrm{S}$.

$$
\chi_{\mathrm{S}}=\frac{1}{2}\left(I_{\mathrm{S}}+A_{\mathrm{S}}\right)=-\frac{1}{2}\left(\varepsilon_{\text {номо }}+\varepsilon_{\text {LUмо }}\right)
$$

where ionization potential $I_{S}$ and electron affinity $A_{\mathrm{S}}$ are approximated by energy of HOMO (Highest Occupied Molecular Orbital) $\varepsilon_{\text {HOMO }}$ and energy of LUMO (Lowest Unoccupied Molecular Orbital) $\varepsilon_{\text {LUMO }}$, respectively. The energies of HOMO and LUMO can be obtained by the first principles DFT calculations. On the other hand, the chemical hardness $\eta_{\mathrm{S}}$ of the species $\mathrm{S}$ is defined by Eq.(3), and it can be also obtained from the energies of HOMO and LUMO.

$$
\begin{aligned}
\eta_{\mathrm{S}} & =\frac{1}{2}\left(\frac{\partial^{2} E_{\mathrm{S}}}{\partial N_{\mathrm{S}}{ }^{2}}\right) \\
& =\frac{1}{2}\left(I_{\mathrm{S}}-A_{\mathrm{S}}\right)=-\frac{1}{2}\left(\varepsilon_{\text {номо }}-\varepsilon_{\text {LUмо }}\right)
\end{aligned}
$$

Now, we consider a chemical reaction between acid A and base $\mathrm{B}$. The reaction proceeds by electron transfer from base B to acid A at the overlap of HOMO and LUMO. The energy change $\Delta E$ associated to the electron transfer, that is the interaction energy, can be described as shown in Eq.(4).

$$
\Delta E=\left(E_{\mathrm{A}}-E_{\mathrm{A}^{0}}\right)+\left(E_{\mathrm{B}}-E_{\mathrm{B}^{0}}\right)=-\frac{1}{2}\left(\chi_{\mathrm{A}^{0}}-\chi_{\mathrm{B}^{0}}\right) \Delta N=-\frac{\left(\chi_{\mathrm{A}^{0}}-\chi_{\mathrm{B}^{0}}\right)^{2}}{4\left(\eta_{\mathrm{A}^{0}}+\eta_{\mathrm{B}^{0}}\right)}
$$


where $\Delta N$ means the number of electron transfer. $E_{\mathrm{A}}, \chi_{\mathrm{A}}$ and $\eta_{\mathrm{A}}$ are energy, electronegativity and hardness for acid $\mathrm{A}$, and the same notions are utilized for base $\mathrm{B}$. Superscript 0 means the quantity of state before chemical reaction. When the interaction energy $\Delta E$ is more negative, $\mathrm{A}$ and $\mathrm{B}$ are more reactionary. In this study, metal ions and in-vivo molecules are set for acid $\mathrm{A}$ and base $\mathrm{B}$, respectively. As the interaction energy $\Delta E$ is more negative for more toxic, bio-compatible elements are distinguished.

\subsection{Search of stable crystal structure for perovskite-type compounds}

Perovskite-type compounds, whish have a chemical formula $\mathrm{ABX}_{3}$, provide well-known examples of displacive phase transitions. They are paraelectric non-polar phase at high temperature and have a cubic crystal structure $(a=c)$ as shown in Fig.2 (a). The cubic crystal structure consists of A cations in the large eightfold coordinated site, B cations in the octahedrally coordinated site, and $\mathrm{X}$ anions at equipoint. The cubic crystal structure often distorts to ferroelectric phase of lower symmetry at decreased temperature, that is a tetragonal crystal structure $(a>c)$ with spontaneous polarization and strain as shown in Fig.2 (b). In ferroelectric distortions caused by structural transition from cubic phase to tetragonal one, $\mathrm{B}$ cations and $\mathrm{X}$ anions have small displacements $\mathrm{Z}_{\mathrm{B}}, \mathrm{Z}_{\mathrm{X} 1}$ and $\mathrm{Z}_{\mathrm{X} 2}$, respectively if unit cell of crystal structure is defined by A cations. The stability of cubic crystal structure can be estimated by geometric essential condition, tolerance factor $t$. If ion radiuses of $\mathrm{A}, \mathrm{B}$ and $\mathrm{X}$ are $r_{\mathrm{A}}, r_{\mathrm{B}}, r_{\mathrm{X}}$ as shown in Fig.3, tolerance factor $t$ can be described as the following equation:

$$
t=\frac{r_{A}+r_{X}}{\sqrt{2}\left(r_{B}+r_{X}\right)}
$$

When tolerance factor $t$ consists in the range from 0.75 to 1.0 , the perovskite-type crystal structure has high stability.

Figure 4 shows the flow chart of stable crystal structure evaluation for perovskite-type compounds by the first principles DFT calculations. At first, stable atomic combinations of $\mathrm{A}, \mathrm{B}$ and $\mathrm{X}$ elements are searched by tolerance factor. And then, a cubic crystal structure with minimum potential energy is derived by structural optimization. Next, potential energy of tetragonal crystal structures is evaluated by employing four parameters, aspect

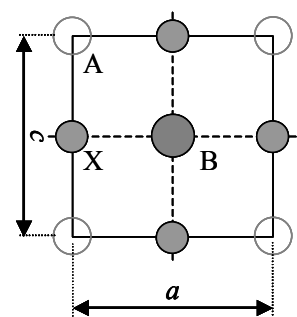

(a) Cubic

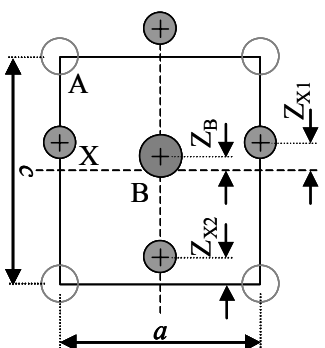

(b) Tetragonal

Fig.2 Perovskite-type cubic and tetragonal crystal structures.

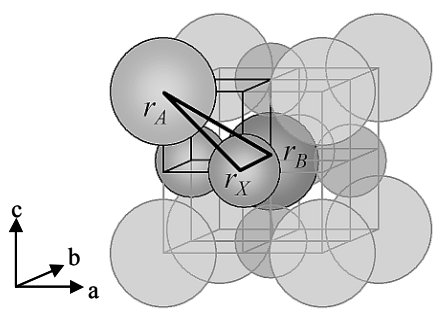

Fig.3 Ion radiuses in perovskite-type cubic crystal structure used for tolerance factor.

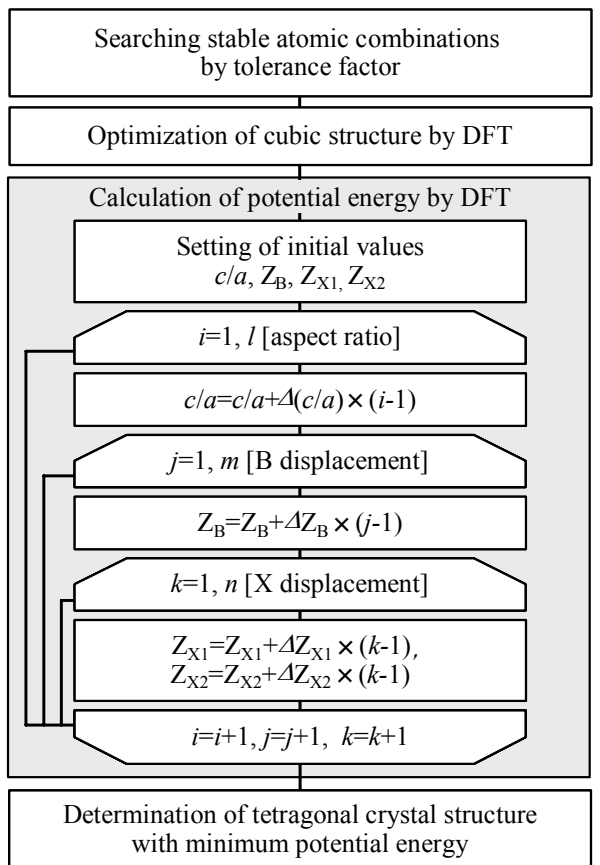

Fig.4 Flow chart of stable crystal structure evaluation for perovskite-type compounds. 
ratio $c / a$ (tetragonality), atomic displacements $Z_{\mathrm{B}}, \mathrm{Z}_{\mathrm{X} 1}$ and $\mathrm{Z}_{\mathrm{X} 2}$. Finally, stable atomic coordinates for a tetragonal crystal structure are obtained by comparison of potential energies.

\subsection{The first principles DFT calculations}

The first principles DFT are employed for energy calculation of molecular orbital of metal ions and in-vivo molecules, crystal structure analysis of perovskite-type compounds. DFT is predicated on two deceptively simple principles ${ }^{(10)}$. One is that the total energy of a system of electrons is unique functional of the electron density as shown in Eq.(6). The other is that the variational minimum of the energy is exactly equivalent to the true ground-state energy.

$$
E=E[\rho(\boldsymbol{r})]
$$

The density $\rho(\boldsymbol{r})$ can be written in terms of the wave functions of the non-interacting electrons.

$$
\rho(\boldsymbol{r})=\sum_{i} \Psi_{i}^{*}(\boldsymbol{r}) \Psi_{i}(\boldsymbol{r})
$$

where $\Psi_{i}(\boldsymbol{r})$ are the $i$-th one-electron wave functions, which satisfy the following rectangular condition.

$$
\int \Psi_{i}^{*}(\boldsymbol{r}) \Psi_{i}(\boldsymbol{r}) d \boldsymbol{r}=\delta_{i j}
$$

where $\delta_{i k}$ means Kronecker's delta.

Now, the energy of a system is treated as shown in Eq.(9).

$$
E=T[\rho(\boldsymbol{r})]+E_{h}[\rho(\boldsymbol{r})]+E_{x c}[\rho(\boldsymbol{r})]
$$

$T$ is the electron kinetic energy and it is defined as the kinetic energy of a system of non-interacting electrons that gives rise to the density $\rho(\boldsymbol{r}) . E_{h}$ is the familiar Hatree coulomb energies of electrons, electrons-cores and cores, and it does not include exchange and correlations effects. $E_{x c}$ is the exchange-correlation functional. When the second variational principle is applied to the energy functional, we obtain the following Kohn-Sham equations ${ }^{(11)}$.

$$
\left\{-\frac{1}{2} \nabla^{2}+V_{\text {eff }}(\boldsymbol{r})\right\} \Psi_{i}(\boldsymbol{r})=\varepsilon_{i} \Psi_{i}(\boldsymbol{r})
$$

where $v_{\text {eff }}(\boldsymbol{r})$ is the effective potential as shown in Eq.(11).

$$
V_{e f f}(\boldsymbol{r})=\frac{\delta}{\delta \rho(\boldsymbol{r})} E[\rho(\boldsymbol{r})]=V_{e x t}(\boldsymbol{r})+V_{h}(\boldsymbol{r})+\frac{\delta E_{x c}[\rho(\boldsymbol{r})]}{\delta \rho(\boldsymbol{r})}
$$

$V_{\text {ext }}(\boldsymbol{r})$ and $V_{h}(\boldsymbol{r})$ are the external term and Hatree coulomb term, respectively. We can obtain self-consistent density $\rho(\boldsymbol{r})$ and wave functions $\Psi_{i}(\boldsymbol{r})$ from iterative solution of Kohn-Sham equations, and then we can calculate potential energy of molecular orbital. Additionally, we can obtain Kohn-Sham solutions of 3D periodic crystal cell by the application of Bloch theorem to the electron wave functions.

\section{Results and Discussions}

\subsection{Bio-compatible elements specified by HSAB principle}

Typical in-vivo molecules, proteins and DNA were utilized for calculation of interaction energy to specify bio-compatible metal elements. There are a great many proteins, and they have large molecule number and complicated molecular structure. It is impossible to consider all kinds and overall structure of proteins into the first principles DFT calculations. Consequently, we focused on typical constituents of proteins and DNA basis, that are 20 amino acids, 5 anions of base, 5 bases terminated by hydrogen and easy-damaged guanine as shown in Table 1, and evaluated the bio-compatibility of metals from interaction energy with them.

To estimate the interaction energy between the above in-vivo molecules and metals, HOMO and LUMO energies of metal ions were calculated by using Gaussian $98^{(12)}$ with 
exception of toxicity and bio-essential elements. We utilized DFT ( Becke \& Lee, Yang, Parr: B3LYP / 6-311++G(d,p) ) for the 3rd or less group elements, and DFT ( B3LYP / LANL2DZ ) for the 4th or more group elements. With the same procedure, we utilized DFT ( B3LYP / 6-311++G(d,p) ) for the in-vivo molecules, and calculated HOMO and LUMO energies. If compared with experimental values of ionization potential and electron affinity, calculational values of HOMO and LUMO energy by using Gaussian 98 were verified to have average errors $3.09 \%$ and $9.07 \%$, respectively.

Table 1 In vivo molecules used for bio-compatible evaluation.

\begin{tabular}{|c|c|}
\hline Anion of base & $\mathrm{CONH}^{-2}, \mathrm{COOH}^{-}, \mathrm{NH}^{2-}, \mathrm{OH}^{-}, \mathrm{SH}^{-}$ \\
\hline $\begin{array}{l}\text { Base terminated } \\
\text { hydrogen }\end{array}$ & $\begin{array}{l}\mathrm{H}-\mathrm{CONH}-\mathrm{H}, \mathrm{H}-\mathrm{COOH}, \mathrm{H}-\mathrm{NH}_{2}, \\
\mathrm{H}-\mathrm{OH}, \mathrm{H}-\mathrm{SH}\end{array}$ \\
\hline Amino acid & $\begin{array}{l}\text { Alanine, Argimine, Asparagine, } \\
\text { Asparatic acid, Cysteine, Glutamic acid, } \\
\text { Glutamine, Glycine, Histdine, Isoleucine, } \\
\text { Leucine, Lysine, Methionine, } \\
\text { Phenylalanine, Proline, Serine, } \\
\text { Threonine, Tryosine, Tryptophan, Valine }\end{array}$ \\
\hline DNA & Guanine \\
\hline
\end{tabular}

Figure 5 shows the interaction energy between in-vivo molecules and metal ions obtained by DFT calculations. The interaction energy depends strongly on kind of metal ions, but it has little influence of in-vivo molecules. Therefore, the average value of interaction energy among in-vivo molecules can be useful to estimate overall bio-compatibility of metal ions. On the other hand, Yamamoto A. ${ }^{(13)}$ employed IC50 value (the half maximal Inhibitory Concentration) which represents the concentration of an inhibitor that is required for $50 \%$ inhibition of cells, and he has been reported the experimental results for cytotoxicity evaluation of 43 metals using murine fibroblasts and osteoblastic cells. To verify the bio-compatibility obtained from DFT calculations, Fig.6 shows the comparison with his cytotoxicity tests. The vertical axis means the average IC50 value obtained from cytotoxicity tests using two cells, and the cytotoxicity is strong at low IC50 value. The horizontal axis shows the interaction energy obtained from DFT calculations, and the chemical reactivity is high if the absolute value of interaction energy is large. Figure 6 excludes toxicity and bio-essential metals from 43 metals, and summarizes 7 common metals between cytotoxicity tests and DFT calculations. As there is good correlation

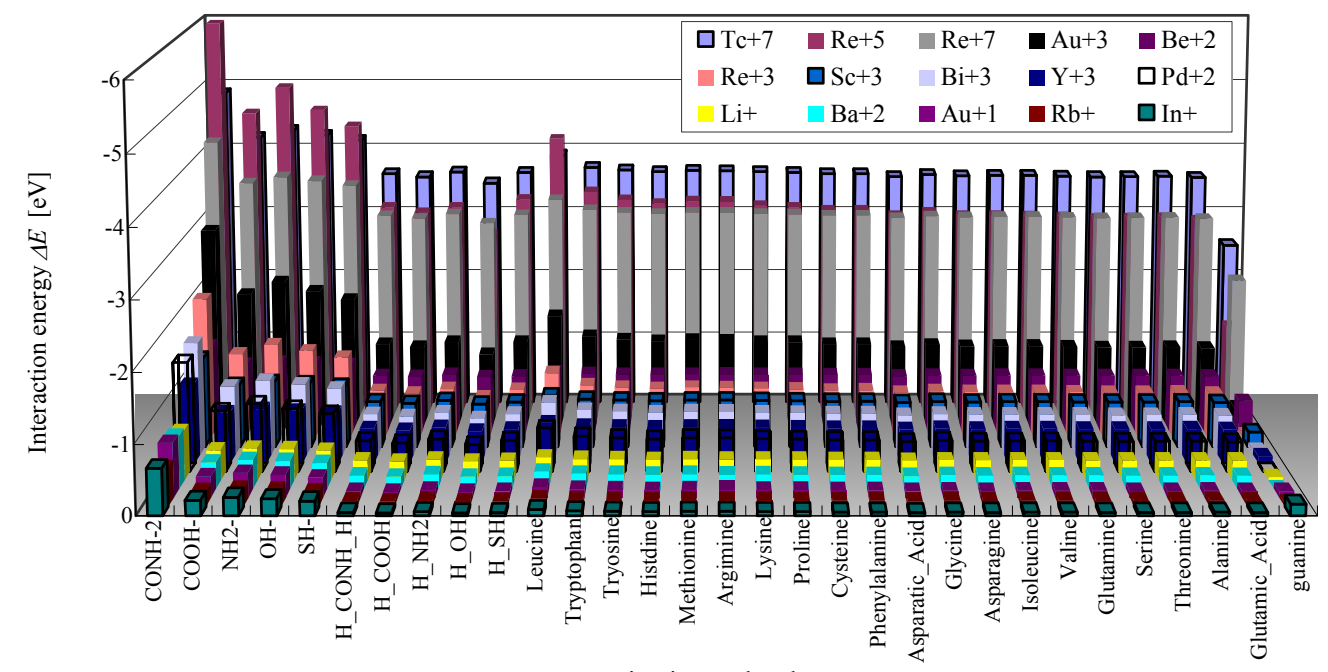

in vivo molecule

Fig.5 Interaction energy between in vivo molecules and metal ions. 
between both results, the interaction energy is an effective indicator enough to estimate bio-compatibility.

The critical IC50 value for bio-materials applied to living body is more than $5.0 \times 10^{-3}$ mol / $l$. If approximated linearly as shown in Fig.6, the interaction energy corresponding to critical IC50 values is more than $-0.3 \mathrm{eV}$. Consequently, metals were distinguished into bio-compatible elements if interaction energy is beyond $-0.3 \mathrm{eV}$. As a result, 6 metals, $\mathrm{Li}^{+}$, $\mathrm{Ba}^{2+}, \mathrm{K}^{+}, \mathrm{Au}^{+}, \mathrm{Rb}^{+}, \mathrm{In}^{+}$were distinguished into bio-compatible elements. We added bio-essential elements and living body-applied elements to bio-compatible elements, and employed them to constituents of bio-compatible piezoelectric material.

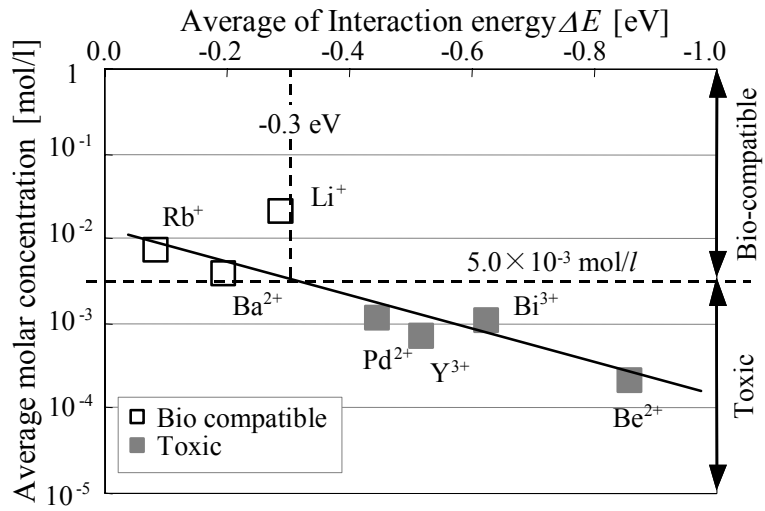

Fig.6 Relationship between experimental result of half maximal inhibitory concentration and calculational result of interaction energy.

\subsection{Crystal structure evaluation of new perovskite-type compounds}

We employed the above bio-compatible elements for $\mathrm{A}$ and $\mathrm{B}$, oxygen for $\mathrm{X}$ in perovskite-type compound $\mathrm{ABX}_{3}$. Table 1 shows stable combinations of $\mathrm{A}, \mathrm{B}$ and $\mathrm{X}$ elements obtained by tolerance factor. In this table, titanium oxides, $\mathrm{BaTiO}_{3}, \mathrm{CaTiO}_{3}$ and $\mathrm{FeTiO}_{3}$ are existing perovskite-type compounds ${ }^{(9)}$. Especially, barium titanate $\mathrm{BaTiO}_{3}$ is one of well-known ferroelectrics with good piezoelectric response, and it was proved to be bio-compatible material in this study. As molybdenum oxides, $\mathrm{BaMoO}_{3}$ and $\mathrm{CaMoO}_{3}$, have no example of fabrication and application, they are considered to be new materials. With regard to some of silicon oxides such as $\mathrm{MgSiO}_{3}$ and $\mathrm{CaSiO}_{3}$, they are perovskite-type

Table 2 Combinations of bio-compatible elements satisfying geometric stable condition defined by tolerance factor.

\begin{tabular}{c|c|c|c|c|c}
\hline $\mathrm{A}$ & $\mathrm{B}$ & $t^{* 1}$ & $t^{* 2}$ & $t^{* 3}$ & Average \\
\hline $\mathrm{Ba}$ & $\mathrm{Ti}$ & 0.9349 & 0.9451 & 0.9970 & 0.9590 \\
$\mathrm{Ca}$ & $\mathrm{Ti}$ & 0.8125 & 0.8244 & 0.8485 & 0.8285 \\
$\mathrm{Fe}$ & $\mathrm{Ti}$ & 0.7309 & 0.7623 & 0.7106 & 0.7346 \\
\hline $\mathrm{Ca}$ & $\mathrm{Si}$ & 0.9337 & 0.9235 & 0.9428 & 0.9333 \\
$\mathrm{Fe}$ & $\mathrm{Si}$ & 0.8399 & 0.8539 & 0.7896 & 0.8278 \\
$\mathrm{Zn}$ & $\mathrm{Si}$ & 0.8360 & 0.8269 & 0.7857 & 0.8162 \\
$\mathrm{Mg}$ & $\mathrm{Si}$ & 0.8009 & 0.8114 & 0.8328 & 0.8150 \\
$\mathrm{Mn}$ & $\mathrm{Si}$ & 0.8595 & 0.8694 & - & 0.8644 \\
\hline $\mathrm{Ba}$ & $\mathrm{Zr}$ & 0.8839 & 0.8727 & 0.9406 & 0.8991 \\
$\mathrm{Ca}$ & $\mathrm{Zr}$ & 0.7682 & 0.7613 & 0.8005 & 0.7766 \\
\hline $\mathrm{Ba}$ & $\mathrm{Mo}$ & - & - & 0.9727 & 0.9727 \\
$\mathrm{Ca}$ & $\mathrm{Mo}$ & - & - & 0.8278 & 0.8278 \\
\hline \multicolumn{7}{|c}{$* 1$} & Calculated by Pauling ion radius \\
\hline \multicolumn{7}{c}{$* 2$} & Calculated by Goldschmidt ion radius
\end{tabular}


minerals existing under high pressure $30 \mathrm{GPa}$ of mantle ${ }^{(14)}$. For an example, $\mathrm{MgSiO}_{3}$ is a mineral called enstatite ${ }^{(15)}$, which is a rhombohedral crystal structure of incomplete ferroelectric phase, it has no good piezoelectricity. However, $\mathrm{MgSiO}_{3}$ can become a new bio-compatible piezoelectric material if made into a tetragonal crystal structure of ferroelectric phase under some suitable conditions. Therefore, we have been analyzed the cubic and tetragonal crystal structures of perovskite-type $\mathrm{MgSiO}_{3}$, and examined the possibility to have good piezoelectricity. CASTEP code ${ }^{(16)}$ released by Accelrys was used for the first principle DFT calculations.

At first, the accuracy of crystal structure analysis was verified with application to a typical perovskite-type $\mathrm{PbTiO}_{3}$. We used the pseudo-potential, linear augmented plane-wave method within the generalized gradient approximation (GGA) for the exchange and correlation interactions between electrons. Figure 7 shows the stable cubic and tetragonal crystal structures obtained from DFT calculations. The lattice constants $a=3.900 \AA$ and

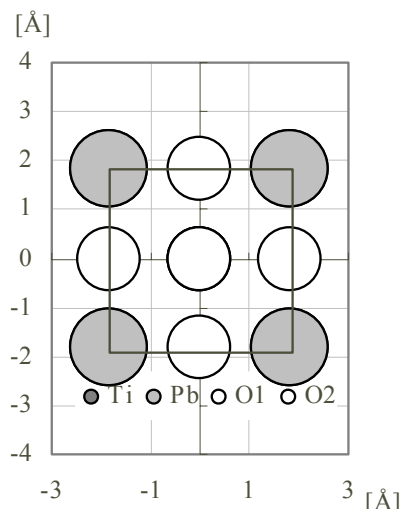

(a) Cubic structure

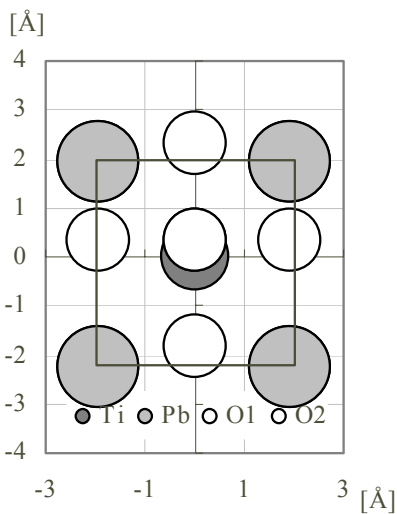

(b) Tetragonal structure

Fig.7 Stable cubic and tetragonal crystal structures of perovskite-type $\mathrm{PbTiO}_{3}$.

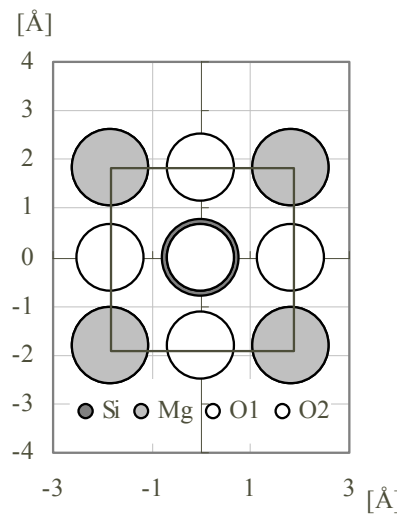

(a) Cubic structure

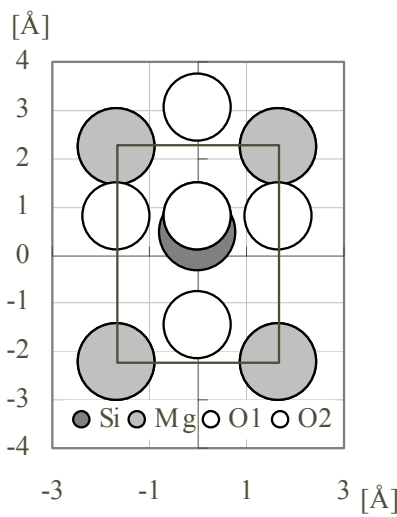

(b) Tetragonal structure

Fig.8 Stable cubic and tetragonal crystal structures of perovskite-type $\mathrm{MgSiO}_{3}$.

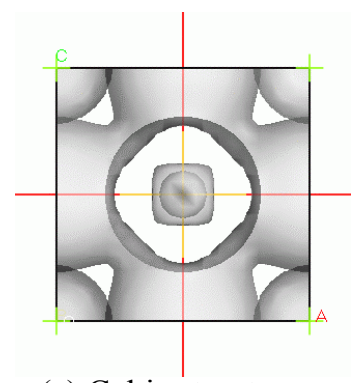

(a) Cubic structure

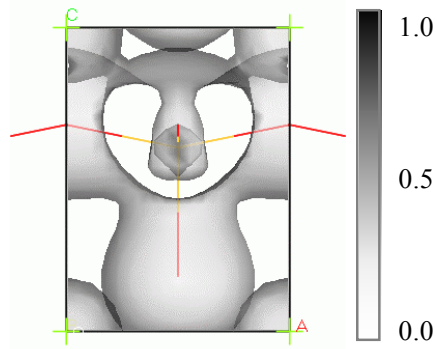

(b) Tetragonal structure

Fig.9 Electron density distribution in cubic and tetragonal crystal structures of perovskite-type $\mathrm{MgSiO}_{3}$. 
$c=4.150 \AA$ estimated from DFT calculations have a good correlation with $a=3.899 \AA$ and $c=4.153 \AA$ measured by $\mathrm{X}$ ray diffraction.

Next, the stable cubic and tetragonal crystal structures of perovskite-type $\mathrm{MgSiO}_{3}$ were analyzed with the same procedure. The initial values of lattice constant was set at $a=c=$ $3.571 \AA$ referring to those of enstatite. The internal coordinates of $\mathrm{Mg}, \mathrm{Si}$ and $\mathrm{O}$ were $\mathrm{Mg}=(0.0,0.0,0.0), \mathrm{Si}=(0.5,0.5,0.5), \mathrm{O}_{1}=(0.5,0.0,0.5)$ and $\mathrm{O}_{2}=(0.5,0.5,0.0)$, respectively. Additionally, energy cutoff was set at $380 \mathrm{eV}$, and k-point was $6 \times 6 \times 6$. Figure 8 (a) shows the estimated stable crystal structure of cubic $\mathrm{MgSiO}_{3}$ with lattice constants $a=c=3.660$ $\AA$. And then, the stable crystal structure of tetragonal $\mathrm{MgSiO}_{3}$ was computed on the basis of cubic crystal structure. The minimum potential structure was searched on the conditions that aspect ratio $c / a$ was changed from 1.00 to 1.54 , atomic displacements $Z_{\mathrm{Si}}$ and $\mathrm{Z}_{\mathrm{O}}\left(=\mathrm{Z}_{\mathrm{O} 1}\right.$ $=Z_{\mathrm{O} 2}$ ) were changed with increments 0.015 and 0.030 , respectively. Figure 8 (b) shows the estimated stable crystal structure of non-symmetric tetragonal $\mathrm{MgSiO}_{3}$ with lattice constants $a=b=3.303 \AA, c=4.493 \AA$. The electron density distribution in cubic and tetragonal crystal structures of perovskite-type $\mathrm{MgSiO}_{3}$ is shown in Fig.9. This figure indicates non-symmetric tetragonal structure has spontaneous polarization $P_{s}=0.508 \mathrm{C} / \mathrm{m}^{2}$ in $c$ axis, while symmetric cubic structure is non polar.

Generally, typical perovskite-type compounds output good piezoelectricity by structural phase transition from paraelectric non-polar phase of cubic structure to ferroelectric phase of tetragonal structure. Figure 10 shows potential energy of perovskite-type $\mathrm{PbTiO}_{3}$ during structural phase transition on the assumption of linear structural change from cubic to tetragonal structure. As potential energy decrease monotonously from cubic to tetragonal structure, perovskite-type $\mathrm{PbTiO}_{3}$ can shows piezoelectric response by spontaneous structural phase transition. With the same procedure, potential energy during structural

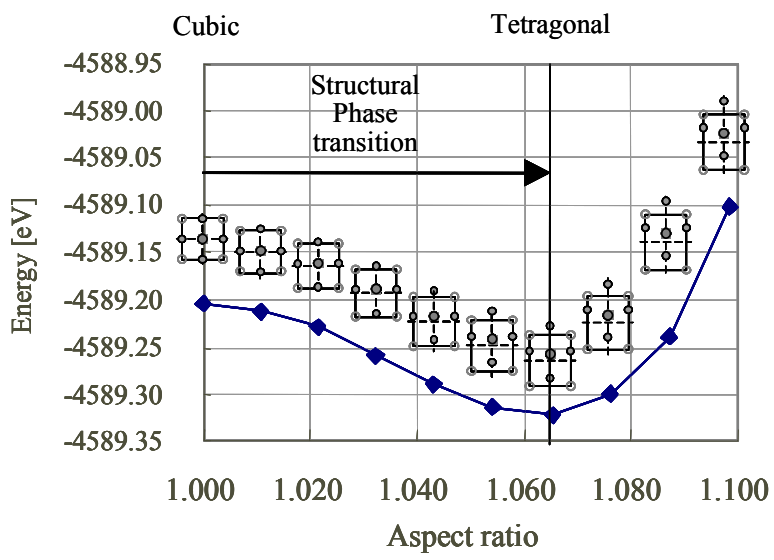

Fig.10 Potential energy of perovskite-type $\mathrm{PbTiO}_{3}$ during structural phase transition.

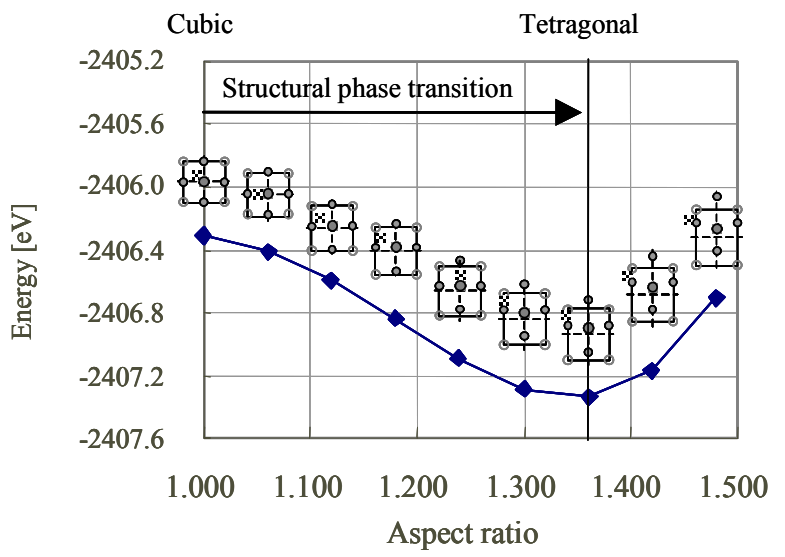

Fig.11 Potential energy of perovskite-type $\mathrm{MgSiO}_{3}$ during structural phase transition. 
phase transition was investigated for perovskite-type $\mathrm{MgSiO}_{3}$. The aspect ratio $c / a$ was changed from 1.00 to 1.54, and the internal coordinates of $\mathrm{Si}$ and $\mathrm{O}$ elements were set by linearization between cubic and tetragonal structures. Figure 11 shows potential energy of perovskite-type $\mathrm{MgSiO}_{3}$ during structural phase transition. The potential energy indicates the similar trend with perovskite-type $\mathrm{PbTiO}_{3}$. Consequently, perovskite-type $\mathrm{MgSiO}_{3}$ can also change spontaneously, and it can be expected to have good piezoelectric response at ferroelectric phase of tetragonal structure.

In addition, we employed helicon wave plasma sputtering, and succeeded to fabricate a perovskite-type $\mathrm{MgSiO}_{3}$ thin film with tetragonal structure at ferroelectric phase by controlling crystal structure ${ }^{(17)}$. Then, we measured the displacement in response to an electric filed by AFM (Atomic Force Microscope), and proved that the perovskite-type $\mathrm{MgSiO}_{3}$ thin film has piezoelectricity.

\section{Conclusion}

In order to derive new piezoelectric materials with bio-compatibility, constituent elements were specified by HSAB method from the viewpoint of interaction energy with in-vivo molecules. Then, the combination of bio-compatible elements was selected to satisfy stable condition defined by tolerance factor to create a perovskite-type crystal structure with good piezoelectric response. As a result, we discovered 7 kinds of new piezoelectric materials. We focused on one of them, $\mathrm{MgSiO}_{3}$ that is known to be a mineral with perovskite-type crystal structure, and analyzed the stable cubic structure at paraelectric non-polar phase and the stable tetragonal structure at ferroelectric phase by first principles DFT. The results indicated that $\mathrm{MgSiO}_{3}$ can change spontaneously to tetragonal structure with high tetragonality and polarization, and that $\mathrm{MgSiO}_{3}$ can present a good piezoelectricity.

This study was supported by Bio-Venture grant from MEXT (the ministry of education, culture, sports, science and technology, Japan).

\section{References}

(1) Roche, D. et al., "Piezoelectric Bimorph Bending Sensor for Shear-Stress Measurement in Fluid Flow", Sensors and Actuators A: Physical, 55, (1996), pp.157-162.

(2) “On Waste Electrical and Electronic Equipment", Official Journal of the European Union, L37 (2003), pp.24-38.

(3) "On The Restriction of The Use of Certain Hazardous Substances in Electrical and Electronic Equipment", Official Journal of the European Union, L37 (2003), pp.19-23.

(4) Stachiotti, M. G. et al., "Electronic Structure and Ferroelectricity in $\mathrm{SrBi}_{2} \mathrm{Ta}_{2} \mathrm{O}_{9}$ ", Physical Review B: Condensed Matter and Materials Physics, 61, (2000), pp.14434-14439.

(5) Kumagai, H., "Piezoelectric Properties and Potential Applications of Potassium Niobate Single Crystals”, Ceramics Japan, 35, (2000), pp.360-362.

(6) Parr R. G. and Pearson G., "Absolute Hardness: Companion Parameter to Absolute Electronegativity”, Journal of American Chemical Society, 105 (1983), pp.7512-7516.

(7) De Proft, F. et al., "An Initio Determination of Substitute Constants in A Density Functiional Theory Formalism: Calculation of Intrinsic Group Electronrgativity, Hardness and Softness", Journal of Physical Chemistry, 97 (1993), pp.1826-1831.

(8) Baeten, A. et al., "Use of the HSAB Principle in Quantitative Structure-Activity Relationships in Toxicological Research: Application to the Genotoxicity of Chlorinated Hydrocarbons", International Journal of Quantum Chemistry, 74 (1999), pp.351-355. 
(9) The Chemical Society of Japan ed., "Perovskite Related Compounds (in Japanese)", (1997), p.7, Japan Scientific Societies Press.

(10) Hohengerg, P. and Kohn, W., "Inhomogeneous Electron Gas", Physical Review, 136, (1964), pp.B864-B871.

(11) Kohn, W. and Sham, L. J., "Self-Consistent Equations Including Exchange and Correlation Effects", Physical Review, 140, (1965), pp.A1133-A1138.

(12) Tazaki, K. trans., "Exploring Chemistry with Electronic Structure Methods (in Japanese)", (1998), Gaussian.

(13) Yamamoto, A. et al., "Cytotoxicity Evaluation of 43 Metal Salts Using Murine Fibroblasts and Osteoblastic Cells", Journal of Biomedical Material Research Part A, 39, (1998), pp.331-340.

(14) Ross, N. L. and Hazen, R. M., "High-Pressure Crystal Chemistry of $\mathrm{MgSiO}_{3}$ Perovskite", Physics and Chemistry of Minerals, 17, (1990), pp.228-237.

(15) Kudoh, Y. et al., "Effect of Pressure on the Crystal Structure of Perovskite-type $\mathrm{MgSiO}_{3}$ ", Physics and Chemistry of Minerals, 14, (1987), pp.350-354.

(16) Segall, M. D. et al., "First-Principles Simulation: Ideas, Illustrations and The CASTEP Code", Journal of Physics: Condensed Matter, 14 (2002), pp.2717-2743.

(17) Japanese Patent Disclosure 2004-355017 (2004). 